Original Research Paper

\title{
QoS Based IPTV over LTE-WLAN Heterogeneous Networks
}

\author{
Mohamed Matoui, Noureddine Moumkine and Abdellah Adib \\ Department of Computer Sciences, Networks, Telecommunications and Multimedia, \\ Faculty of Sciences and Techniques of Mohammedia, Morocco
}

\author{
Article history \\ Received: 22-02-2019 \\ Revised: 03-04-2019 \\ Accepted: 18-04-2019 \\ Corresponding Author: \\ Mohamed Matoui \\ Department of Computer \\ Sciences, Networks, \\ Telecommunications and \\ Multimedia, Faculty of \\ Sciences and Techniques of \\ Mohammedia, Morocco \\ Email: matoui.mohamed@gmail.com
}

\begin{abstract}
Heterogeneous networks required IP Multimedia Subsystem (IMS) technologies to perform their control signaling and improve their communication services. However, the highest IPTV-QoS cannot be performed using IMS only as it cannot differentiate between IPTV video components. In this paper, we implement our IPTV-flow label technique in LTE-WLAN heterogeneous systems that used to improve QoS by providing IPTV sub traffic priority according to the network administrator policy. The proposed architecture has been implemented and analyzed using Opnet Modeler 17.5 software. The produced results demonstrate that IPTV users receive high definition video data with a variation in quantity in conformity with data priority by using Opnet 17.5 software.
\end{abstract}

Keywords: IPv6, Heterogeneous Networks, LTE, WLAN, QoS

\section{Introduction}

The composition of new telecommunication architectures has become indispensable, in a more converged environment that is controlled by the emergence of "triple play". Such openness will stop all the features of fixed/mobile heterogeneity. Internet Protocol (IP) is currently representing an essential convergence of Next Generation Network (NGN) in the attempt to find an inter-service and inter-network matching solution. Terminals have become increasingly integrated and prevalent in the world of converged IP. Providing multimedia clients with adaptable needs and operative efficient services conditioning on the operating environment at the time of service provision is one of the most important and main issues facing the telecom operators. The different devices connected via a heterogeneous access network allow end customers to access some multimedia services. The final costumer receives a differential quality of the IPTV traffic according to resources of the acquisition device and network performance. It should be noted that other constraints, such as the sensitivity of multimedia traffic to packet loss, delay and jitter, are added to that of quality. This is encouraging us to conceive solutions to improve the QoS by acting on the software compositions of service delivery, i.e., those authenticating and marking users. The negotiation of the current state of the reception of the multimedia content as well as the quality of service with the entity's transmitting service will be an asset to the operator on the horizon to provide the traffic with an acceptable quality of service. The translation of this strategy of convergence in the telecommunications industry appears in IMS which is a system that allows the convergence of fixed, wireless and mobile networks in a typical network architecture where all services are working in an all-IP environment. IMS suppose that the operator controls the media consumption by multimedia sessions using Session Initiation Protocol (SIP) to guarantee the desired QoS and convergence between services. Recent experiences reveal that the IMS system still does not solve some problems related to the differentiation of the various IPTV video constituent. The IMS-based IPTV in its current form doesn't take into consideration that the IPTV traffic comprises three sub-components or the delicacy of the linear television delay. With the object of controlling and guarantying the QoS in IMS infrastructure, several methods have appeared namely: Service Level Monitoring and Management (SLM\&M) approach (Raouyane et al., 2011), 3rd Generation Partnership Project (3GPP) approach (Siddiqui et al., 2009) and, Internet Engineering Task Force (IETF) approach (Zamora and Przybysz, 2011). The DiffServ (Differentiated Services) model is used by all the three suggestions for QoS management. A thorough study reveals that traffic classification assumed by these approaches suffer from various issues. The classification of traffic uses three classes: voice, data and video. In connection with IPTV, we declare that traffic can be classified into three sub-traffics:

- BC (BroadCast) that permit the transfer of realtime video

- VoD (Video on Demand) that comprise a library that permits the user to select and view a video 
- PVR (Personal Video Recorder) that allow users to record the received stream

DiffServ model performs the processing of these three sorts of flows similar. The variance in sensitivity to QoS parameters necessitates a reclassification among them. Our contribution goal is to cure this problem. A plethora of types of research has been working on improving IPTV services QoS. Li and Chen (2017) argue IPTV mobility over a wireless cellular network utilizing spectrum allocation technique. This provides better IPTV services by maintaining a good quality of voice service. Li (2017), a new queue model that consider adaptive modulation and coding has been in a large extent the solution to IPTV data difficulties such as dropping, blocking and bandwidth. IPTV seamless handover has been fulfilled thanks to the terms of using load-conscious and physical restraint in a wireless LAN. (Fard and Rahbar, 2016). This technique permits the user to select the next wireless LAN to reach depending on its power, congestion and bit error rate. Tsolkas et al. (2017), the authors furnish a detailed guide to standardized and cutting edge quality evaluation models. They also single out and typify the parametric Quality of Experience (QoE) form for the most of famous service types (i.e., VoIP, video streaming, online video, etc.), indicating the Key Performance Indicators (KPIs) and important configuration parameters per type. Huang et al. (2017) suggest a QoE forecast based on data for the IPTV service. Especially, they establish QoE to assess IPTV user experience in data-driven approach from scratch and develop a particular QoE model based on an Artificial Neural Network (ANN) (Huang et al., 2017). In (Solera et al., 2018), the evaluating of QoE of video streaming service over LTE is described. Different network states, in the testbed, are configured by arranging settings of network emulator rested on the results realized by a system-level LTE simulator. In (Alsaffar el al., 2017), Authors furnish a new technique to diminish tunneling charges by permitting multimedia flow to be sent from various Micro data center as well as Mega data center by employing their proper particular addresses to perform tunneling to convey multimedia content. Kumar et al. (2018) suggest a new rentable wireless architecture consisting of a mix of wireless access technologies (WIFI, satellite and LTE/5G Millimeter Wave (mmWave) overlay connections), for sending live TV services. In our previous work (Matoui et al., 2017a), we introduced in details the new Per-Hop Behavior (PHB) that classify and distinguish IPTV sub traffics by employing the IPv6 Flow Label field. The suggested PHB will make possible prioritization of sub traffics in accordance with the applied QoS network policy. We have previously tested our approach on a fixed network (Matoui et al., $2017 \mathrm{a}$ ) and then on a mobile one in case of moving user in one cell (Matoui et al., 2017b) and in case of stable and moving users in three different cells (Matoui et al., 2018a; 2018b). In this study, we start with a description of the IMS network and IPTV. We equally introduced our new mechanism that serves to improve the QoS of linear television traffic and applied it to the LTE network to enhance the data transfer. Our new QoS optimization algorithm has been explained in section 2 . That exhibit is reliant on how to prioritize IPTV sub-traffic utilizing the IPv6 Flow Label field and how to generate new classes of services. In Section 3, we present our simulation network and the scenario elaborated of the heterogeneous LTE-WLAN-IMS-Based IPTV by using OPNET 17.5 Modeler. Section 4 exposes the outputs analysis of the proposed scenario of the networks methods. Finally, Section 5 discusses the conclusion and our prospects in improving that field.

\section{Improving QoS employing a New Definition of IPv6 Flow Label}

The ability of the network to afford the user requirements upon using IPTV service putting into account the main parameters such as traffic losses, delay, video jitter and quality is the core of the definition of QoS in our network. Two main QoS models were proposed by Internet Engineering Task Force (IETF): Integrated Services (IntServ) and DiffServ (3GPP, 2011), (Bhattarakosol, 2010) (Sambath et al., 2016). The difference between these models is illustrated further (Jiang, 2012). IPv6 Flow Label (FL) has been used along with IMS system to ameliorate QoS for IPTV services, throughout transmission.

\section{IPv6 Flow Label and Quality of Service}

IPv6 FL is a 20-bits field introduced in the IPv6 header. This field can be applied to label packets of another similar packet flow or an aggregation of flows (Deering and Hinden, 2017). Various proposals have been suggested to the IETF to employ this field in order to improve QoS on the internet $(\mathrm{Hu}$ and Carpenter, 2011). Certain researchers have proposed using this field to send the delay, bandwidth and buffer requirements. Other ones have recommended utilizing this field to send the used port number and the transport protocol (Prakash, 2004). Other approaches have been introduced (Conta and Rijahalme, 2001) but actually, none of them have been standardized. However, there is a hybrid proposal that takes into account the progressives approaches and applies them to the DiffServ model (Hu and Carpenter, 2011). This model has recommended using the first 3 bits of the IPv6 FL field to show the methods adopted and kept the remaining 17-bits 
parameter $\mathrm{d}$ to each particular approach. Table 1 sums up this hybrid approach.

\section{Enhancing of IPTV Linear Television}

To optimize QoS for IPTV services throughout transmission, IPv6 FL has been utilized with IMS system. The IMS-Based IPTV was not restricted to the supply of essential services of IPTV, but it expands to other services like 'quadruple play' services and other more advanced ones as Flow Label to enable the user to solicit a unique process for its real-time traffic flow (Bhattarakosol, 2010). To warrant the best appearance for scenes and modulation process, Variable data rate has been assigned for video traffic (Lloret et al., 2012), (Farmer et al., 2016). But that attribution causes same encapsulation problems and control for video flow in a DiffServ network due to the difficulty of designing utmost inter-video traffic limit. Also, DiffServ core routers encounter saturation issue when sending significant traffic with a high priority of Expedited Forwarding (EF) Per-Hop Behavior (PHB). Because of the expansion of real-time data traffic expecting a delay in the queue due to the use of narrow queues attributed to EF PHB technique. It equally produces tardy filtering of video packets which causes their deletion. At the level of dropping process, EF packets will be processed at the periphery of the DiffServ domain depending on their importance in the GROUPE of Picture (GOP) video (Leghroudi et al., 2011). The reject priority for PHBs in AF (Assured Forwarding) is often fulfilled based on Weighted Random Early Detection (WRED). User classification according to their loyalty has been incorporated into IMS-based IPTV by employing the enhanced Telecom Operation Map (eTOM) (Raouyane et al., 2011). Using it, the network administrator proceeds the differentiation between recipient based packages. For instance, if a user is classified as"GOLD," scoring inter users produce another factor to differentiate between the same user with the same classification to affect the currency of transmission. But in case of congestion, the DiffServ model will be used by routers to return to the deletion process. As previously stated, we can classify IPTV video data stream into three principal flows: BC, VoD and PVR. So it will be handled the same in best effort particularly in case of traffic congestion (Sabry et al. 2016). That will cause traffic latency for sensitive video traffic to loss rate and delay. So the necessity for reclassification mechanism between IPTV packets became requisite to reduce that delay and packet losses for, especially BC users. To ameliorate QoS for IPTV services throughout transmission, IPv6 FL has been used along with the IMS system. To reach that request, we suggest new priority removal PHBs for IPTV traffic that differentiates between user data based on their priority (Matoui et al., 2017a). As the IPV4 header's Type of Service (ToS) field is limited to one byte, this technique will map Differentiated Service Code Point (DSCP) values into the IPv6 flow label field, which will differentiate the different IPTV flow by using more bits while remaining compatible with the DiffServ approach. The IPv6 flow label field will thus have the values shown in Table 2.

Up-to-date IPv6 Flow Label Values and as the value of the DSCP field for the EF class is equal to 101110, the IPv6 flow label field can be defined as shown in Table 3.

Where $\alpha, \beta$ are the bits used to distinguish the video traffic intra-IPTV. As IPTV packets have the same value of the DSCP field, then we will reserve the bits 10 and 11 in the IPv6 FL to a reclassification intraIPTV. The rest of 9-bits will be kept for future use. We give the name DSCP-FL to the first 11 bits of the IPv6 FL field. These new Flow Label values are mapped to PHBs that are characterized by a high priority, jitter, low loss rate and, delay like of that of the existing EF PHB. Indeed, three IPTV packets belonging successively under BC traffic, VoD and PVR will be subjected to processing represented through the algorithm in Fig. 1.

Table 1: The representation of the first 3 bits of flow label value type of the used approach

\begin{tabular}{ll}
\hline 000 & Default \\
\hline 001 & A random number is employed \\
& to define the Flow Label. \\
& IntServ \\
010 & Diff-Serv \\
11 & A format that contains the port \\
& number and the protocol in the \\
& Flow Label is used. \\
& A new definition described \\
101 & in (Jee et al., 2002). \\
& Reserved for future use. \\
110 & Reserved for future use \\
\hline
\end{tabular}

Table 2: New IPv6 flow label values

\begin{tabular}{|c|c|c|c|c|c|c|c|c|c|c|c|c|c|c|c|c|c|c|c|}
\hline$\underline{0}$ & 1 & 2 & 3 & 4 & 5 & 6 & 7 & 8 & 9 & 0 & 1 & 2 & 3 & 4 & 5 & 6 & 7 & 8 & 9 \\
\hline 0 & 1 & 1 & DSCP & & & & & & $\alpha$ & $\beta$ & & ed I & futu & & & & & & \\
\hline
\end{tabular}

Table 3: New detailed IPv6 Flow Label Values

\begin{tabular}{llllllllllllllllll}
\hline 0 & 1 & 2 & 3 & 4 & 5 & 6 & 7 & 8 & 9 & 0 & 1 & 2 & 3 & 4 & 5 & 6 & 7 \\
\hline 0 & 1 & 1 & 1 & 0 & 1 & 1 & 1 & 0 & $\alpha$ & $\beta$ & Reserved for future use & & & \\
\hline
\end{tabular}


Table 4: Flow Label value with Highest Priority Level of Suppression

\begin{tabular}{|c|c|c|c|c|c|c|c|c|c|c|c|c|c|c|c|c|c|c|}
\hline \multirow[t]{2}{*}{0} & 1 & 2 & 3 & 4 & 5 & 6 & 7 & 8 & 9 & 0 & 1 & 2 & 3 & 4 & 5 & 6 & 7 & 8 \\
\hline & 1 & 1 & 1 & 0 & 1 & 1 & 1 & 0 & 0 & 1 & & $\mathrm{~d}$ fo & re & & & & & \\
\hline
\end{tabular}

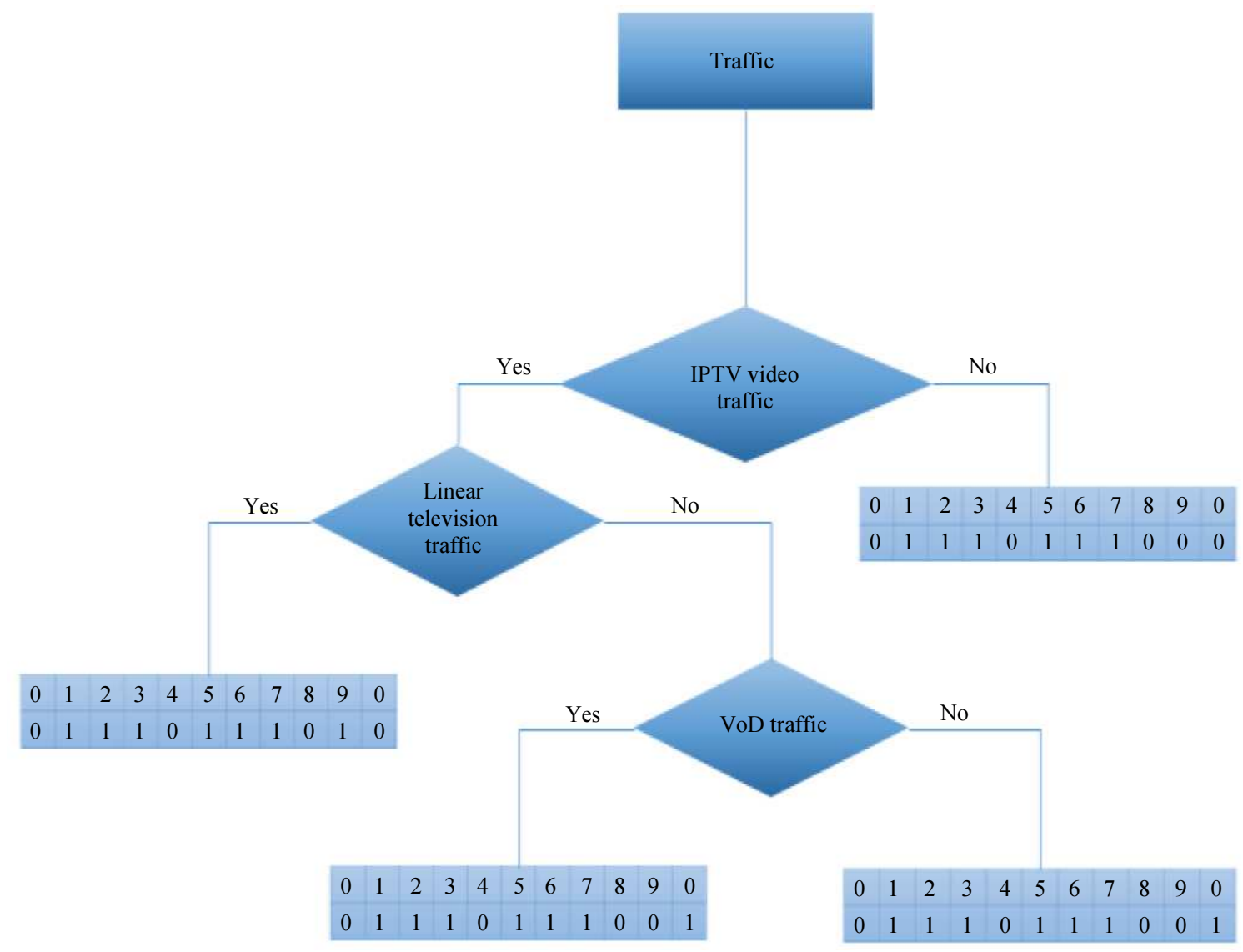

Fig. 1: Our suggested algorithm to differentiate intra-IPTV Traffic

In saturation case, Diffserv routers will remove packets with low-level priority; in the explained case, it is to be the ones whose DSCP-FL field has a value equal to 01110111001 as shown in Table 4.

\section{Implementation Scenario}

The objective of our simulation is to increase the data received by $\mathrm{BC}, \mathrm{VoD}$ and PVR user sequentially and reduce the latency that confronts the $\mathrm{BC}$ traffic especially. Using OPNET 17.5 software, we realized our suggested technique in a heterogeneous LTE-WLAN network. The main idea in the proposed framework architecture is to realize IMS-Based FL IPTV components in the heterogeneous network.

As the IMS-SIP server does not belong to Opnet modules, a new modulated task application module has been developed.MIP-SIP is used for centralized session's control. IMS-MIP-SIP has been used to grantee fast and continuous IPTV service during vertical handover between WLAN and LTE networks. To grantee this task, newly developed modules has been implemented inside Riverbed Modeler as the used server doesn't implement in the exists modules. We defined IMS, MIP-SIP and IPTV servers in the OPNET library. Also, we implement the tasks used during the authentications. After that, we compare the results of our simulation for each user in two cases; while moving inside the LTE network and when moving in the WLAN network.

We have also developed a personalized application in the proposed structure that integrates user registration into the IMS network and IPTV service session establishment. The first instance without applying our approach. The second one conforms to applying FL and WFQ (DSCP Based) QoS technique. Fig. 2 introduces three principal elements of the used architecture. The first element includes the IMS network, the second one contains three servers that substitute the IPTV data center responsible for delivering different types of multimedia contents (PVR, VoD and BC). 


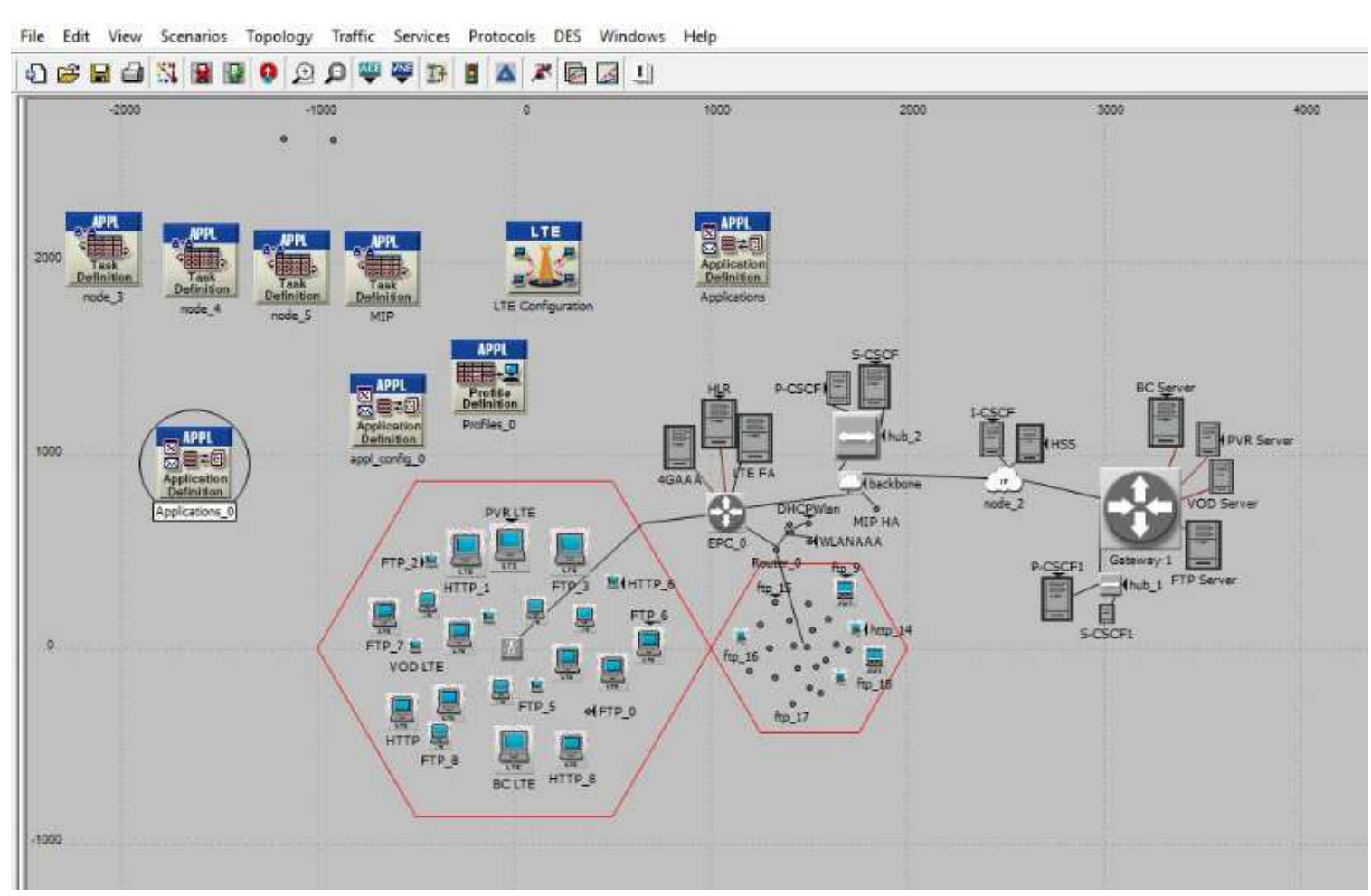

Fig. 2: Moving IPTV user in the LTE-WLAN heterogeneous network

The final one is the personal receiver that receives data from the sender. There are also 10 FTP and 10 HTTP users that send data in parallel IPTV users. We should note that we parallel the conclusions of our suggested scenario upon using and disusing our proposed approach to evaluate the QoS parameters. The three video servers (PVR, BC and VoD) deliver high-definition video and that after the user execute IMS authentication proceedings. We apply our technique by modifying the IPv6 Flow Label for both the servers and the routers inside our network. We also configure QoS parameters in the used routers to guarantee high performance in our network. As described before, the IMS-level registration and session initiation must be proceeded to authenticate the user inside the IMS network before the user initiated the IPTV connection. To proceed vertical handover without any call drop, we choose MIP-SIP protocol that provides minimum handover time.

\section{Performance Analysis}

In this section, we make overall performance analysis for the collected results; the gathered results are packet end-to-end delay and traffic dropped. Then we contrast the performance of the three users (BC, VoD and PVR) in case of using and disusing Flow Label QoS. First, we will explain the results of the user when moving inside the LTE network then when the user made a handover to WLAN network and move inside the network. In each case, we confront the performance of the three users (BC, VoD and PVR) in case of using and without using our technique. Our proposed FL QoS show high performance for BC user.

\section{User Moves Inside LTE Network}

In this scenario, we compare the QoS parameters for the three users when applying our technique. These last ones move at the same speed in the same cell. Our suggested Flow Label QoS show high performance for BC user.

\section{Traffic Dropped}

Figure 3 shows that the three users sent the same quantity of data, while Fig. 4 exhibit that the quantity of data received by $\mathrm{BC}$ user is greater than both $\mathrm{VoD}$ and PVR users as the first one has the highest priority then VoD and PVR users. Figure 5 and 6 shows that $\mathrm{BC}$ and $\mathrm{VoD}$ users received a higher quantity of data when using FL QoS. On the other hand, the quantity of data received by the PVR user diminish when using our approach as shown in Fig. 7 as our technique has assigned the lowest priority to the PVR user flow when transferring data. So, when the three users request the services at the same time, the lowest priority in data transfer will be given to PVR. 


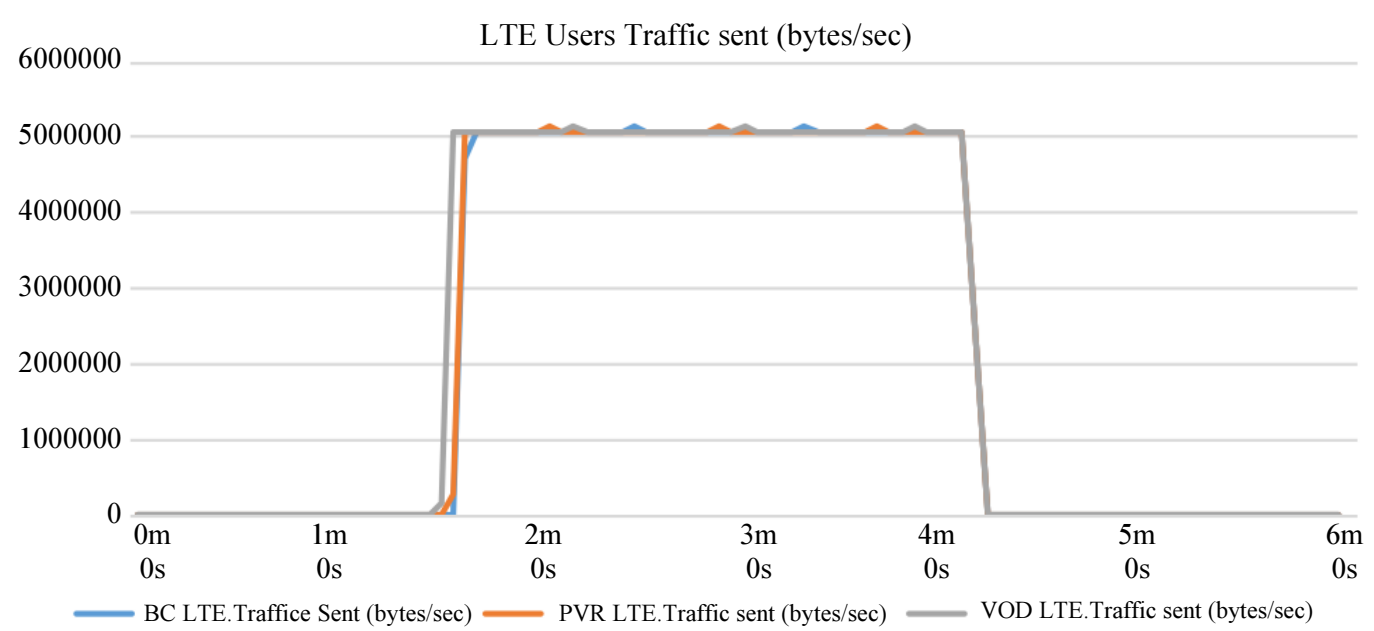

Fig. 3: Traffic sent (bytes/sec)

6000000

LTE Users. Traffic-received (bytes/sec)

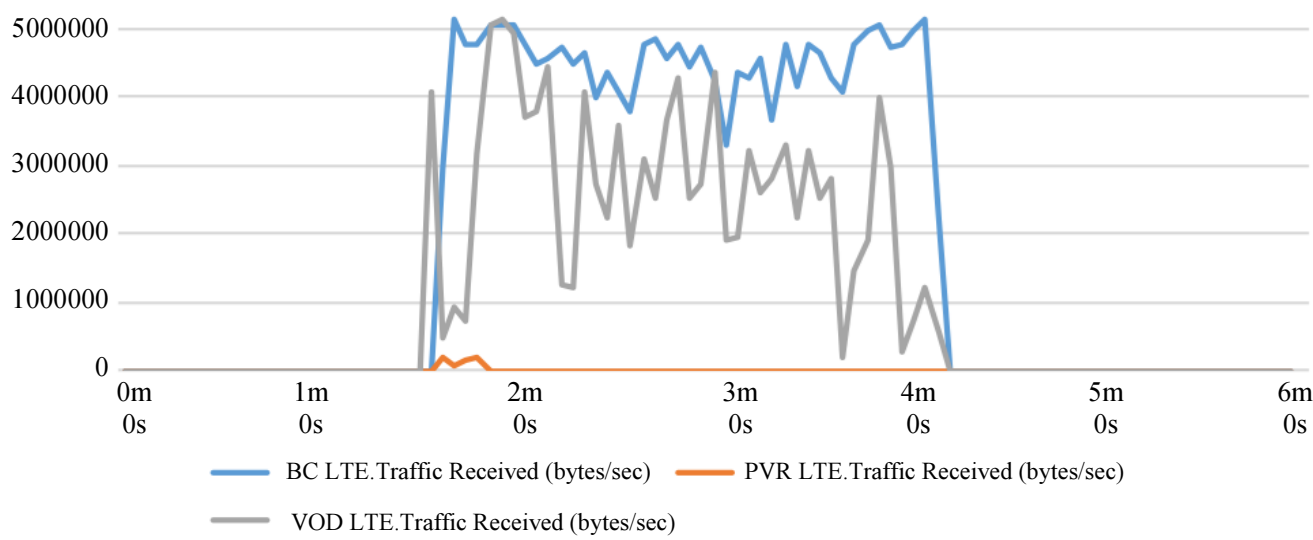

Fig. 4: Traffic received using flow label QoS (bytes/sec)

6000000

BC LTE Traffic received (bytes/sec)

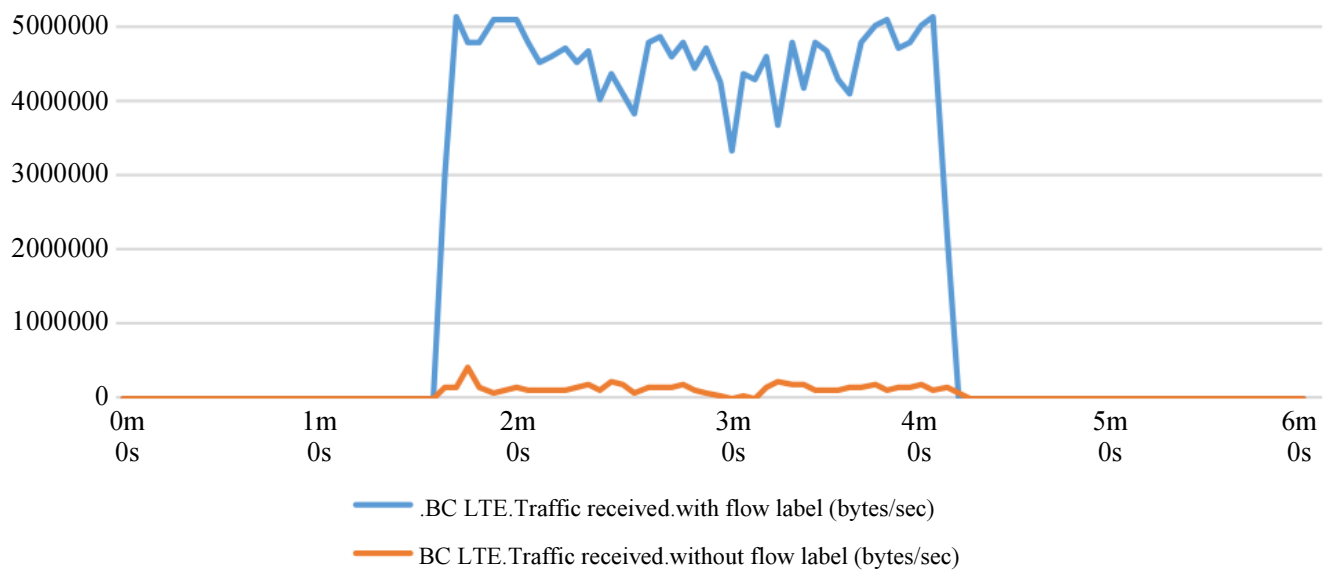

Fig. 5: Traffic received by BC user (bytes/sec) 


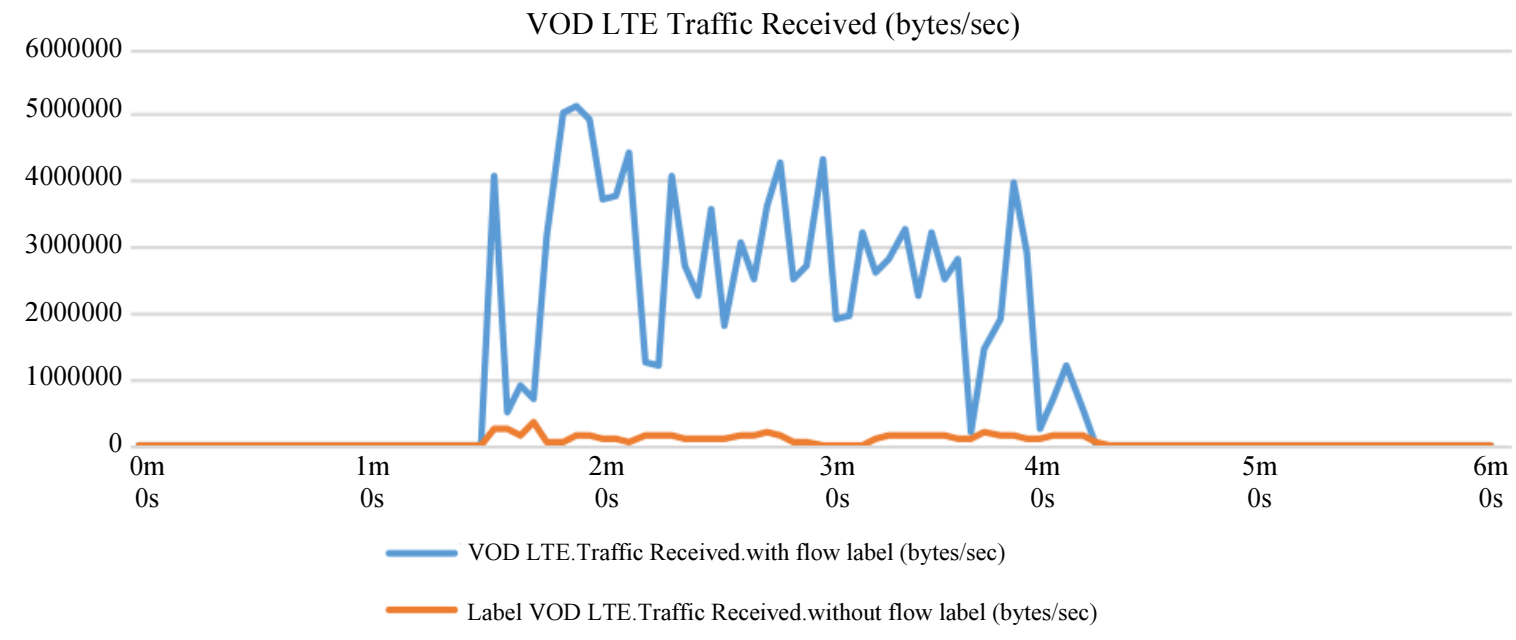

Fig. 6: Traffic received by VoD user (bytes/s)

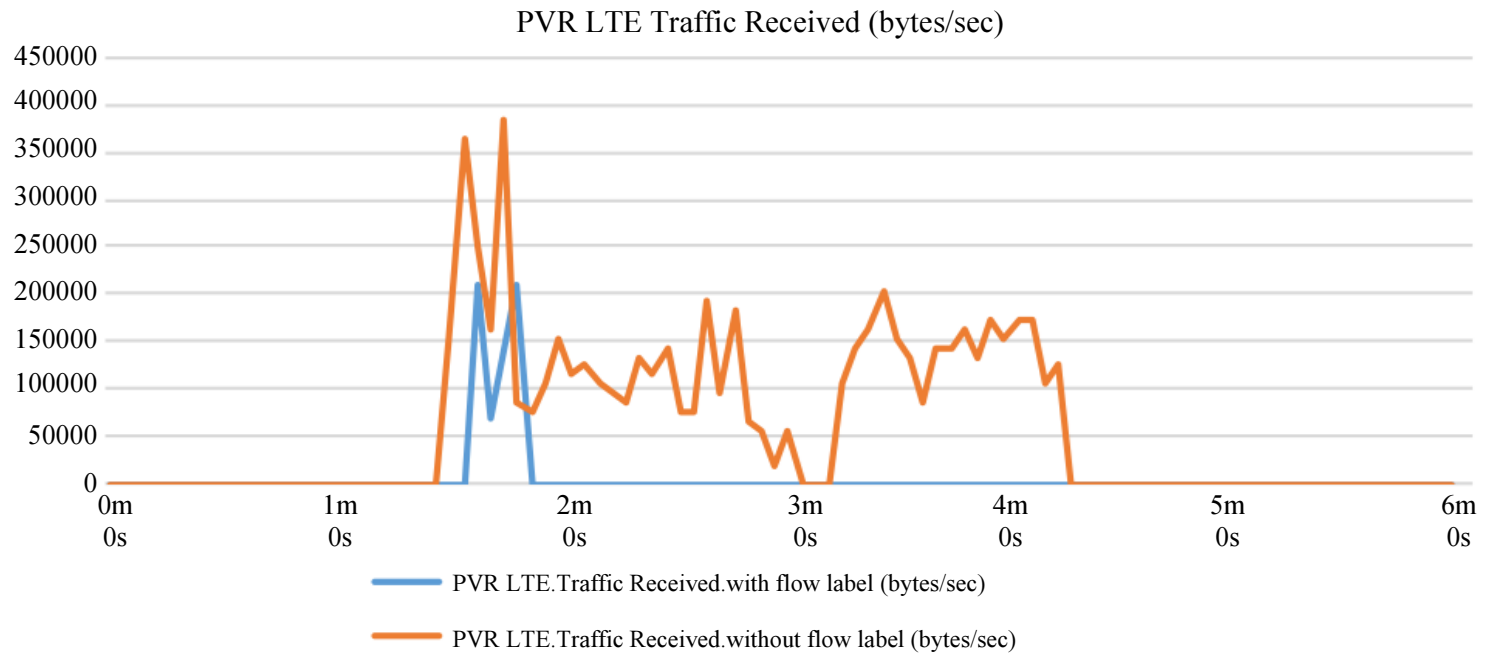

Fig. 7: Traffic received by PVR user (bytes/s)

LTE User.with flow label.Packet.End-to-End-Delay (sec)

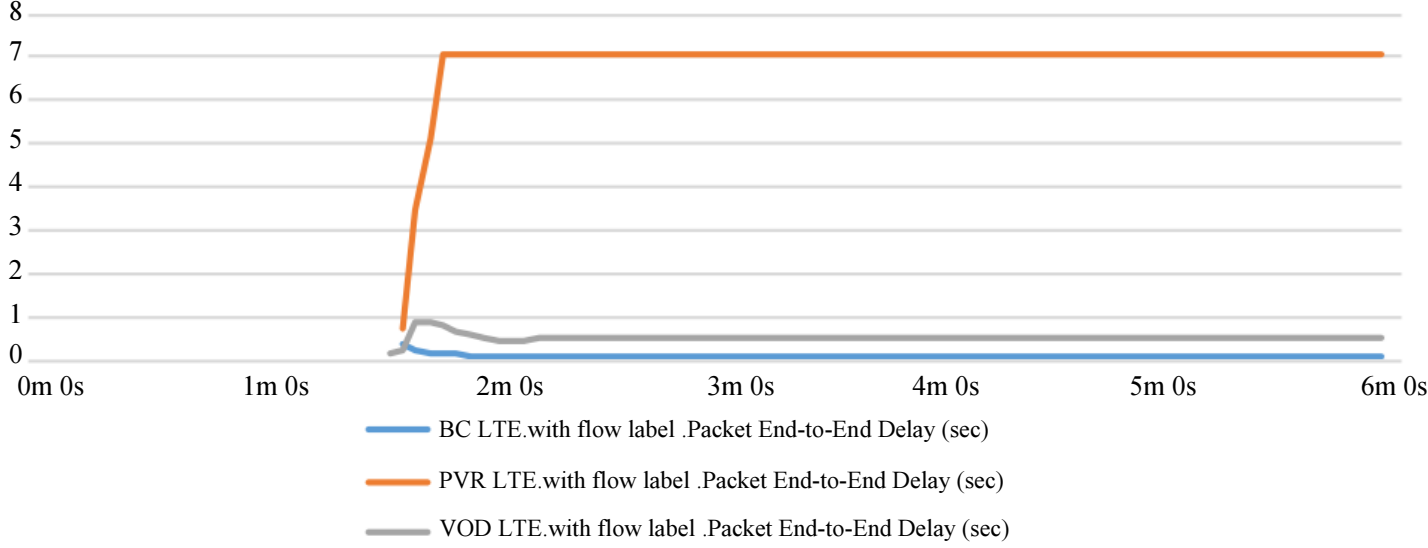

Fig. 8: End-to-end delay (sec) 
BC-LTE.Packet.End-to-End-Delay

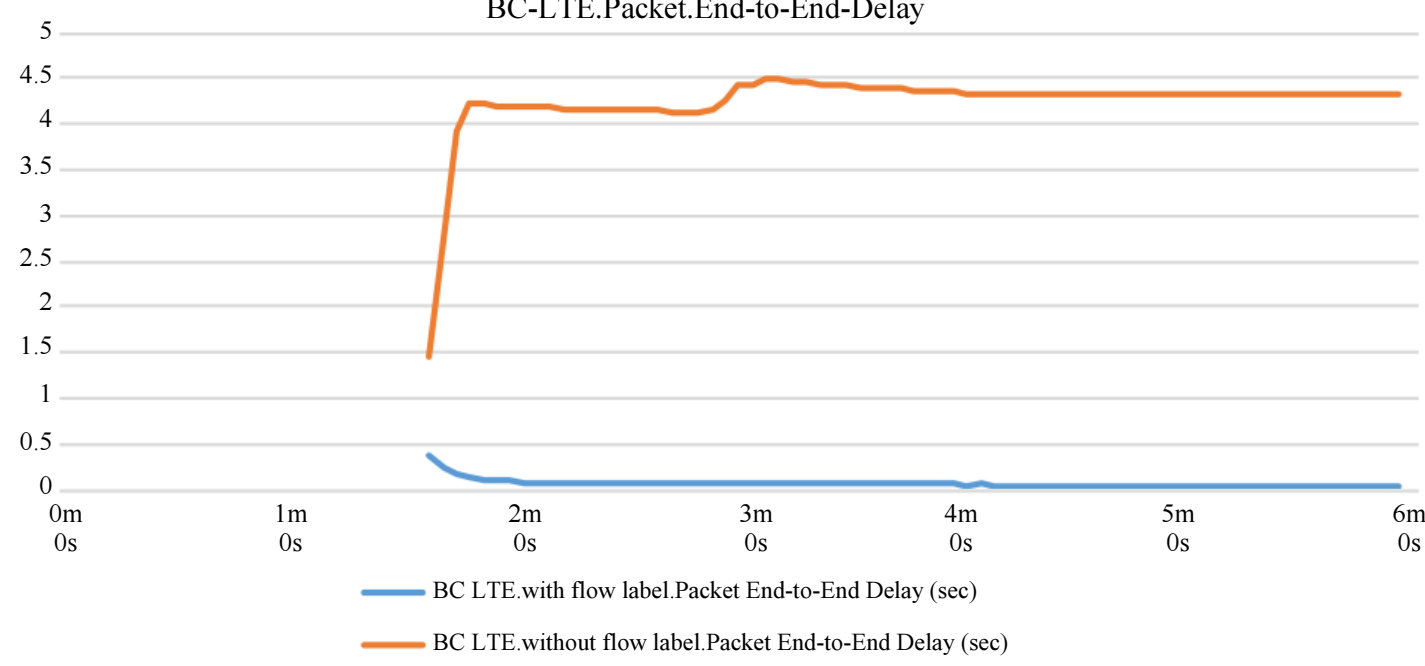

Fig. 9: End-to-end delay taken by BC user (sec)

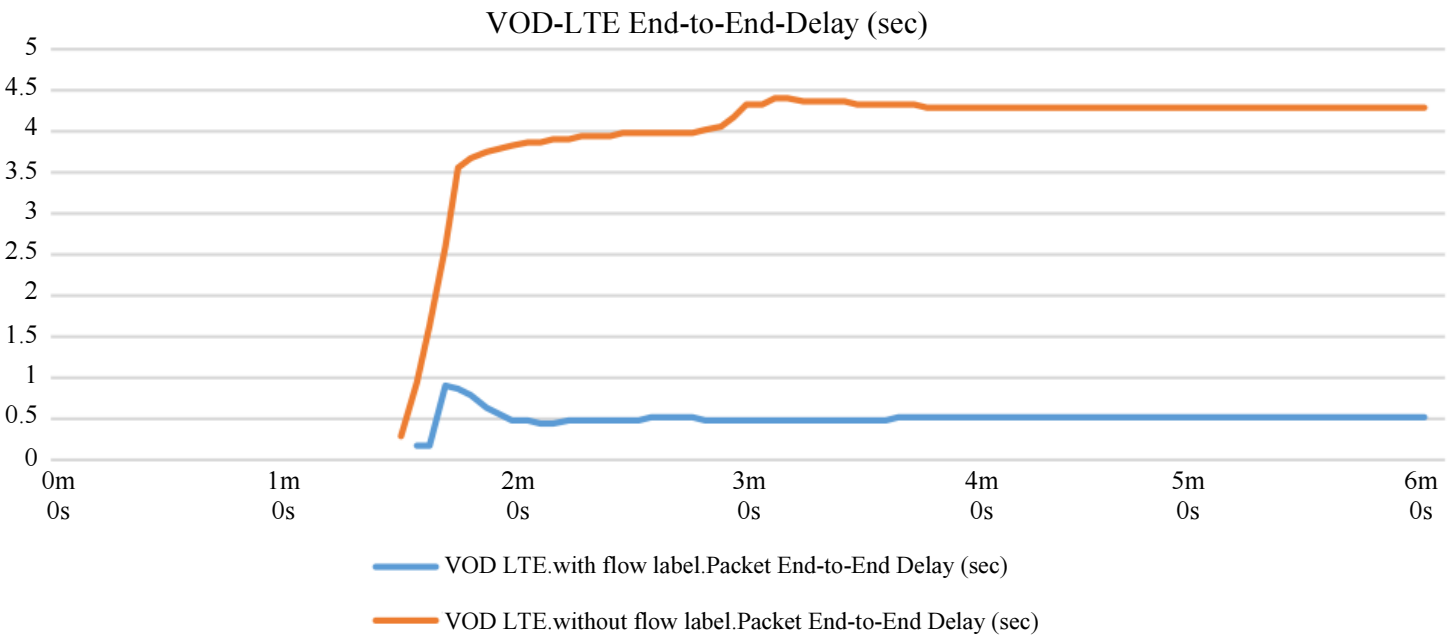

Fig. 10: End-to-end delay taken by VoD user (sec)

PVR-LTE.Packet-End-to-End-Delay (sec)

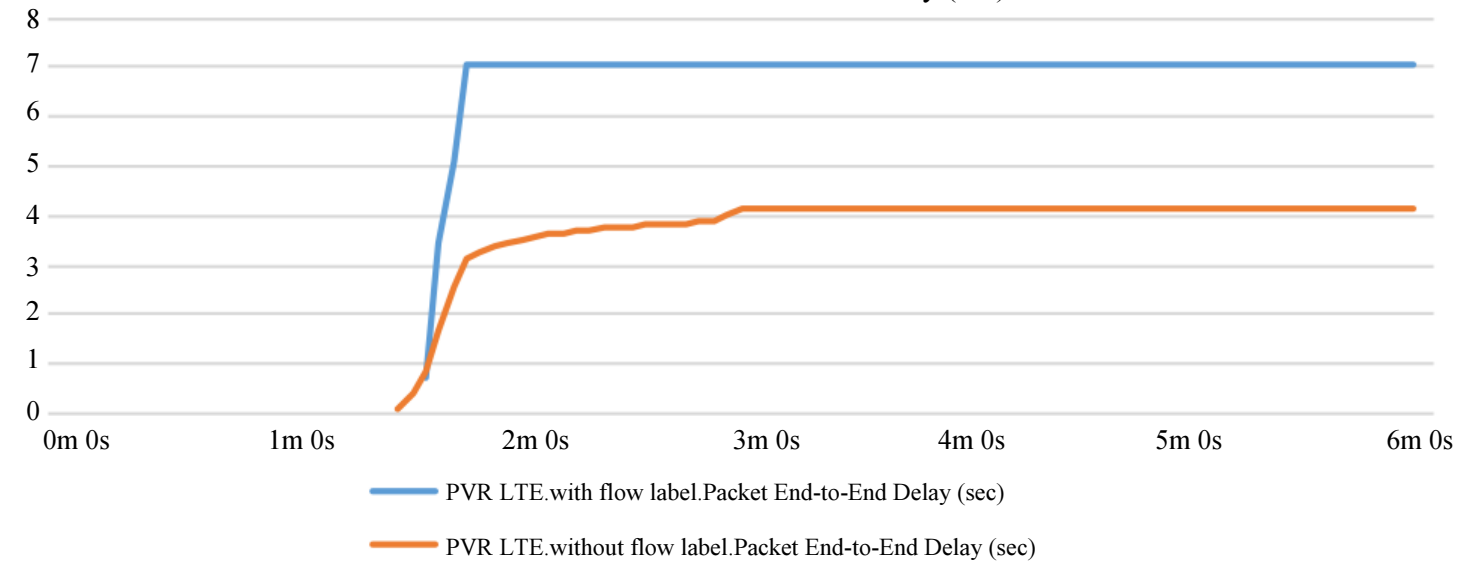

Fig. 11: End-to-end delay taken by PVR user (sec) 


\section{End-to-End Delay}

End-to-end delay can be defined as the time taken by the packets to move from the server to the user. Figure 8 show that End-to-end delay taken by the BC user is the lowest when using FL QoS. This is due to the priority affected in our technique in the proposed scenario. As expressed in Fig. 9 the BC packet delay decreases in case of applying our suggested approach. In the other hand, Fig. 10 and 11 show that the delay of VoD and PVR users raises in case of utilizing our FL QoS technique. The delay of VoD user rises in the small rate while PVR delay rises significantly because, in case of congestion, our technique begins with the processing of $\mathrm{BC}$ user flows and then the VoD user flows before those of the PVR user.

\section{User Moves inside WLAN Network}

\section{Traffic Dropped}

Figure 12 show that the three users sent the same quantity of data. In spite of that, $\mathrm{BC}$ user received the highest quantity of data as shown in Fig. 13, due to its highest priority.

The traffic received by $\mathrm{BC}$ user rises with gigabytes when using our Flow Label QoS technique as shown in Fig. 14. Figure 15, shows that amount of traffic received for VOD user decreased with small amount. As the cell contains other users who send data, so when applying our mechanism IPTV users will have the priority among all of them. Unlike that, the decrease with large amount as shown in Fig. 16. Taking into account that when the user moves, the distance between him and BS varies so the amount of data varies.

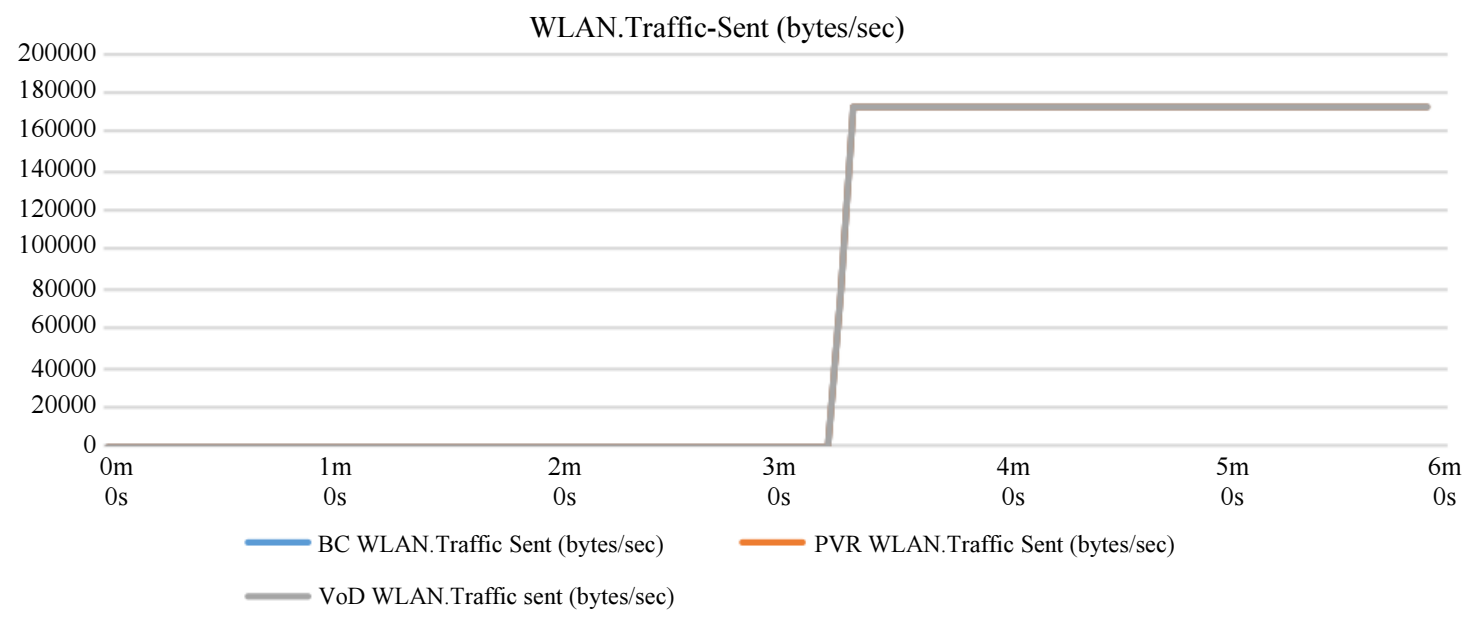

Fig. 12: Traffic sent (bytes/sec)

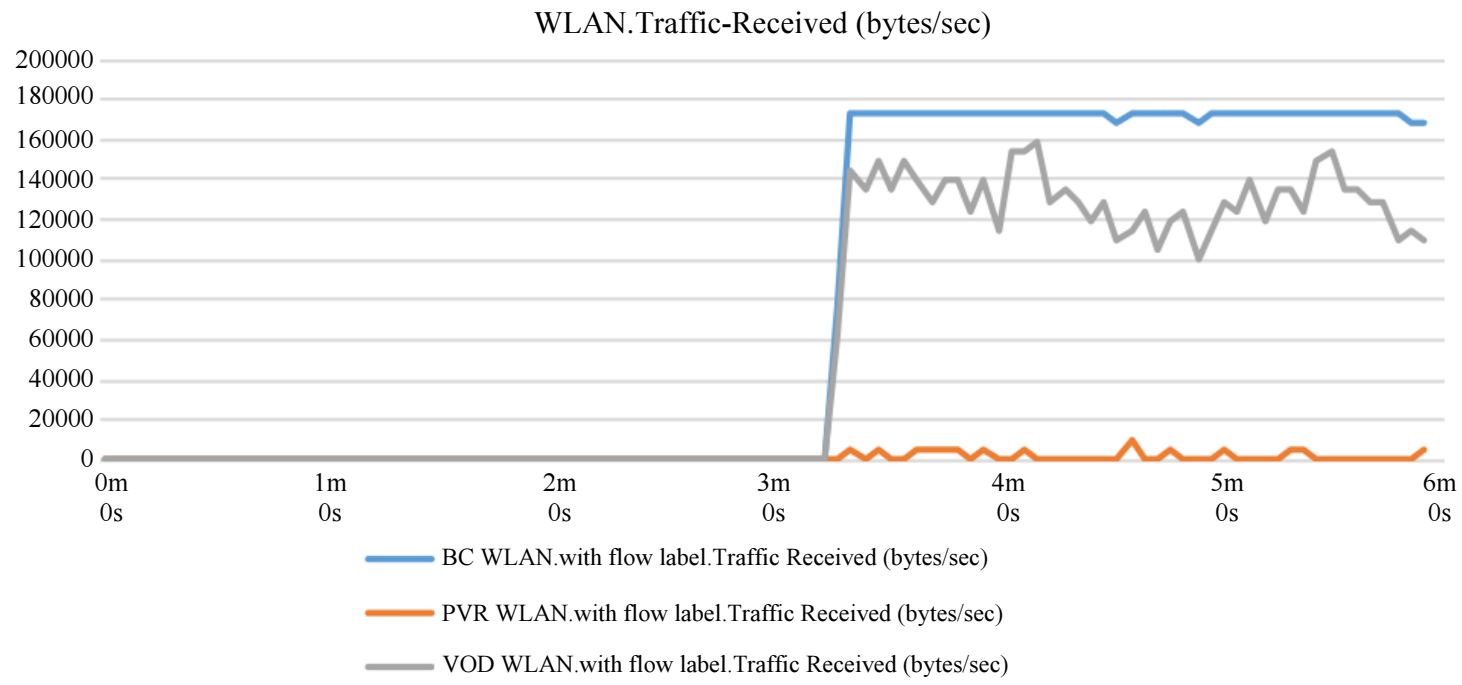

Fig. 13: Traffic received using flow label QoS (bytes/sec) 


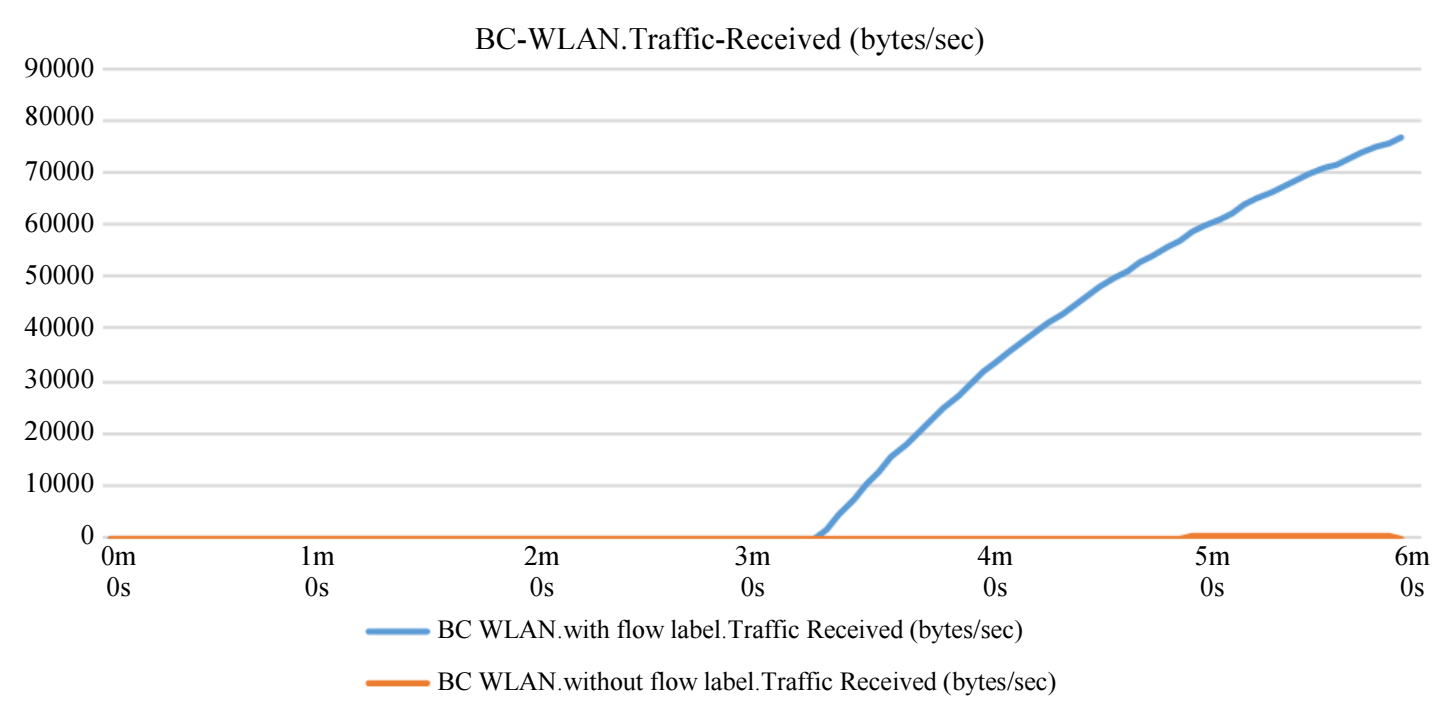

Fig. 14: Traffic received by BC user (bytes/sec)

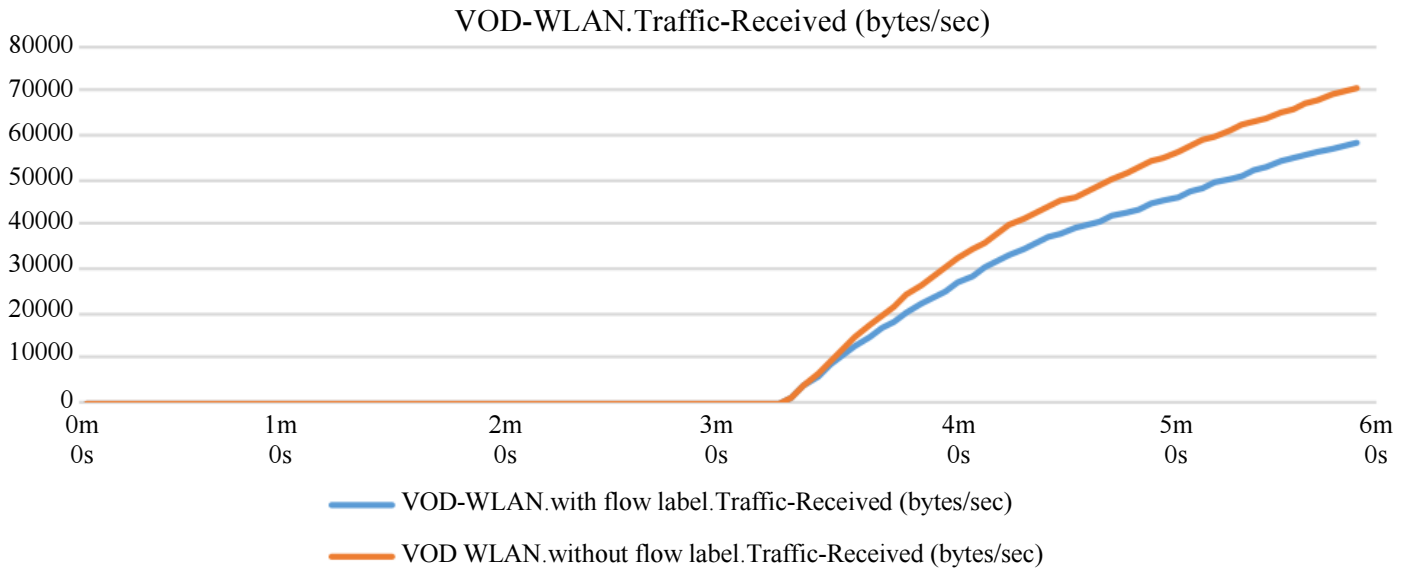

Fig. 15: Traffic received by VoD user (bytes/sec)

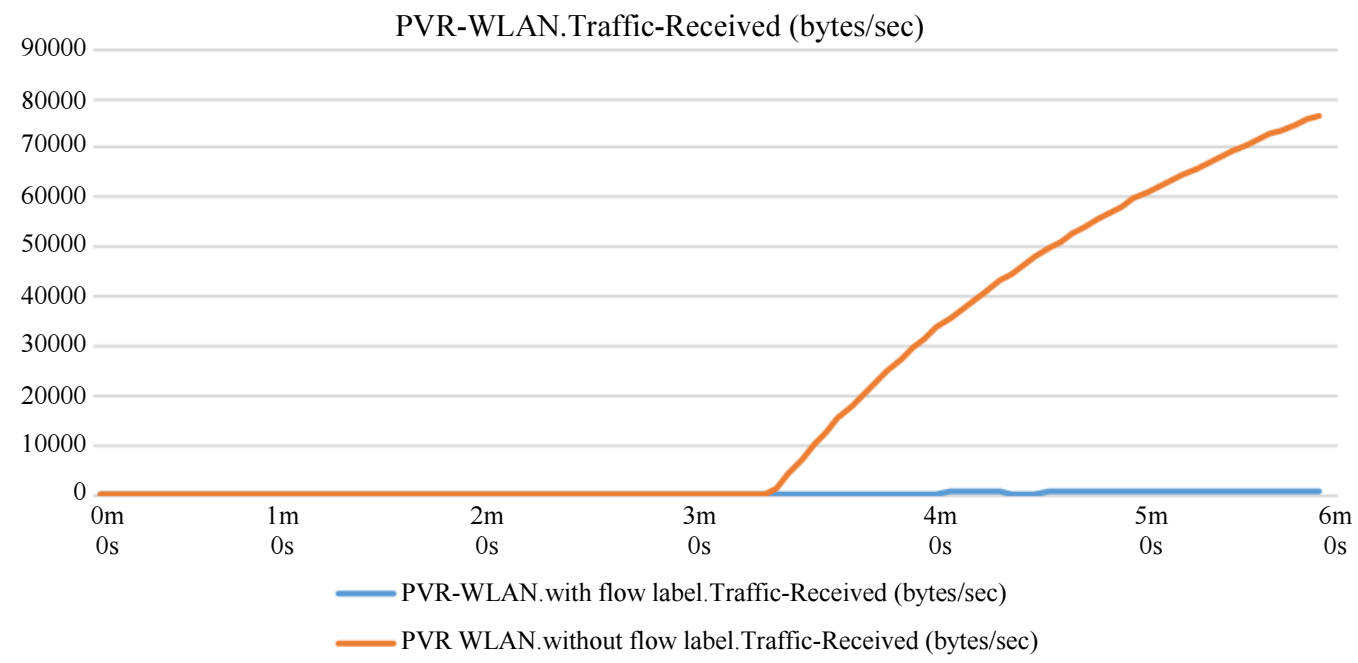

Fig. 16: Traffic received by PVR user (bytes/sec) 


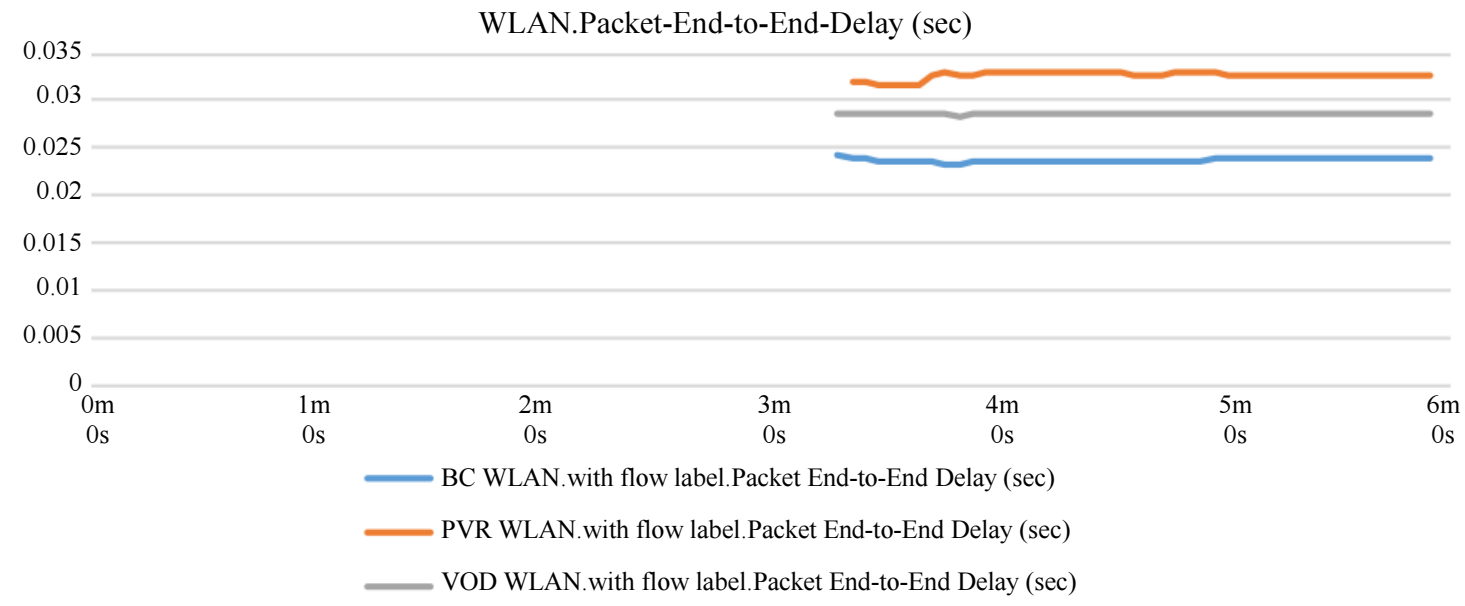

Fig. 17: End-to-end delay (sec)

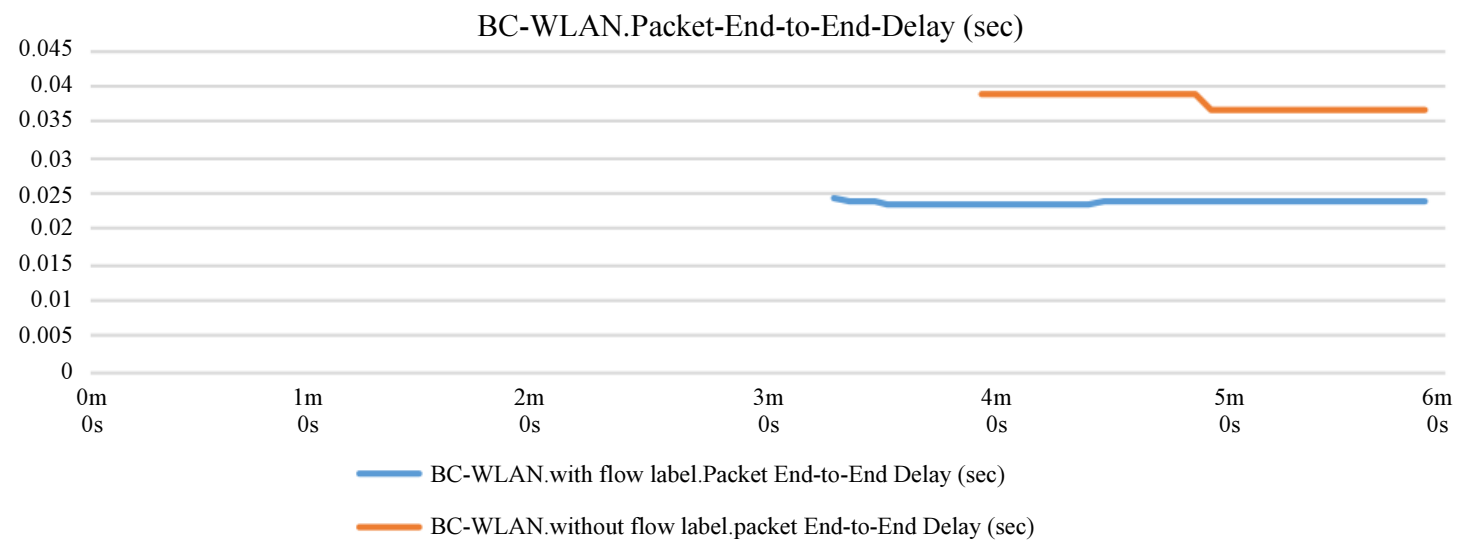

Fig. 18: End-to-end delay taken by $\mathrm{BC}$ user (sec)

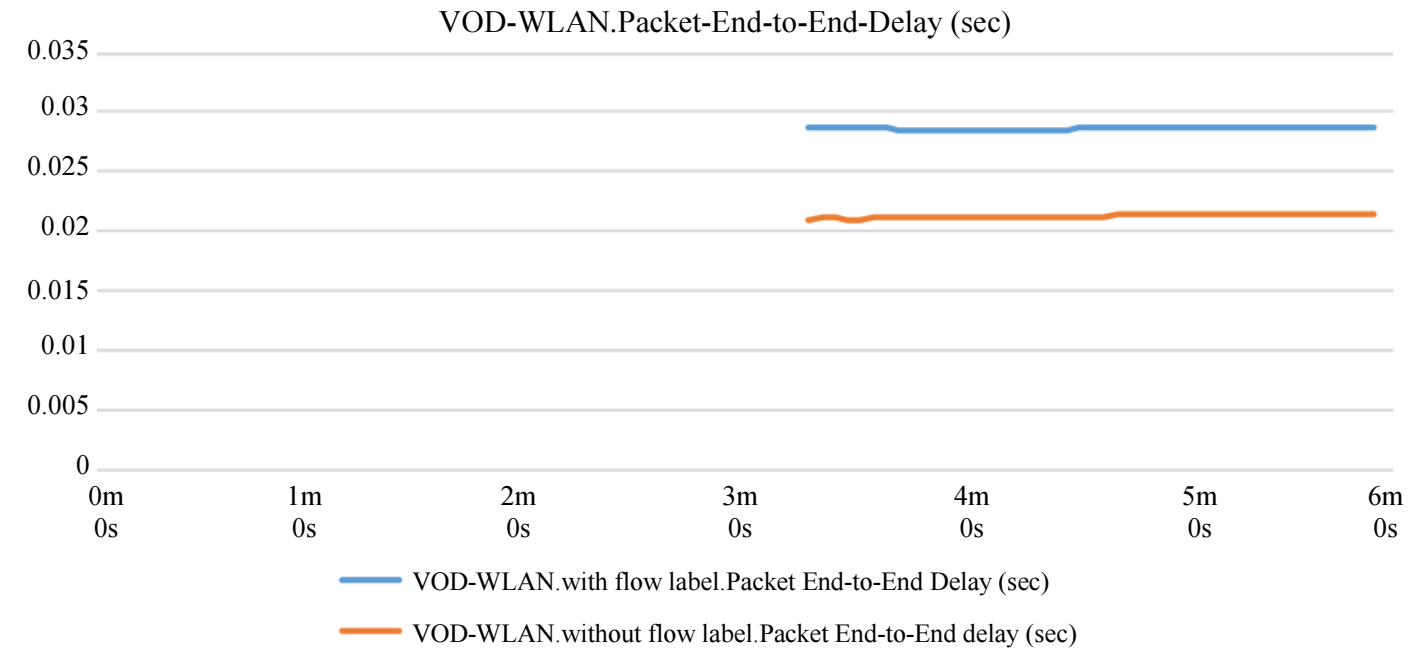

Fig. 19: End-to-end delay taken by $\mathrm{VoD}$ user (sec) 


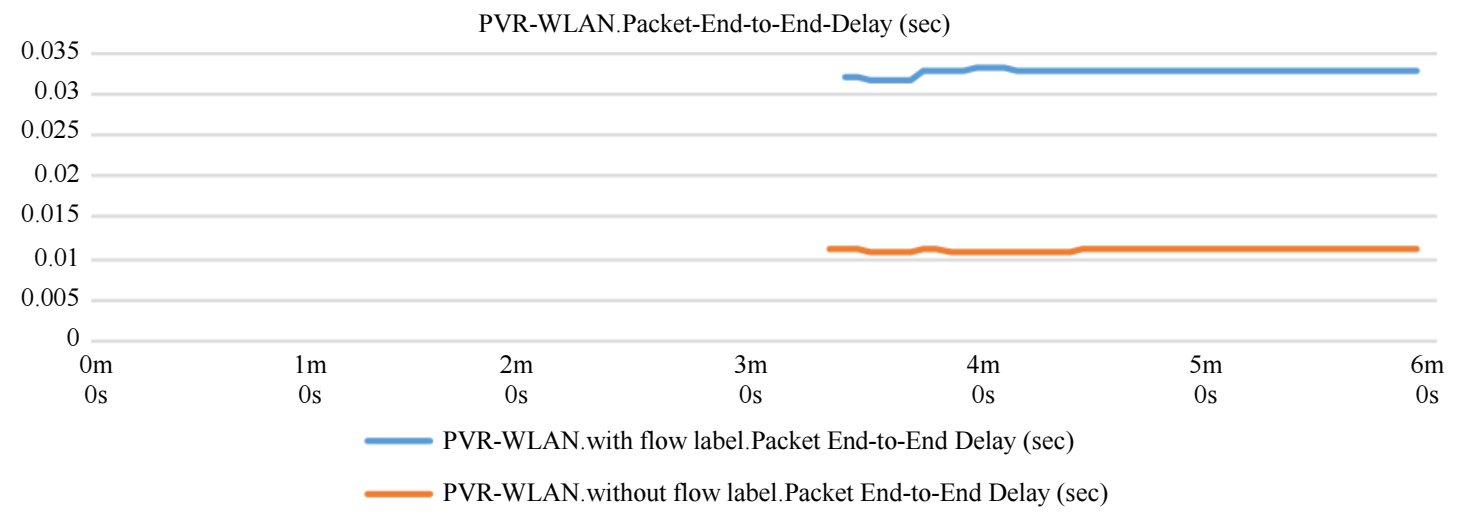

Fig. 20: End-to-end delay taken by PVR user (sec)

\section{End-to-End Delay}

Figure 17 show that the $\mathrm{BC}$ user has the lowest delay while the PVR user has the highest one. This is due to the priority applied in our suggested technique in the proposed scenario. Figure. 18 shows that the BC packet delay decreases in case of applying our FL QoS technique. In the other hand, Fig. 19 and 20 show that the delay of VoD and PVR users rise in case of using our approach. The delay of $\mathrm{VoD}$ user raises in the small rate while PVR delay increases with high rate because our technique prioritizes $\mathrm{BC}$ traffic at the expense of other VoD and PVR traffic.

\section{Discussion}

In this scenario, we notice that $\mathrm{BC}$ flow delay and jitter have been minimized as a aresult of our new classification technique due to the sensitivity of this subtraffics to latency. Our results show that our approach of reclassification and differentiation of the packets enable routers to optimize linear television traffic to the detriment of VoD and PVR flows. This scenario is just a demonstration of the effectiveness of our reclassification method. In reality, it is up to the administrator to choose the highest priority traffic depending on the policy followed by the service provider. The differentiation of IPTV flow allowed us to set up PHBs particular to each IPTV video flow. The results of the simulations show the added value of our classification algorithm in improving the quality of service of broadcast video.

\section{Conclusion and Perspectives}

To conclude there has been a lot of research in the previous years that have been trying to enhance the QoS of IPTV services that regard real-time traffic; principally traffic losses, jitter and latency. None of them consider the trouble of classification of IPTV sub traffic and the distinction between the $\mathrm{BC}, \mathrm{VoD}$ and PVR flows. To fix this problem, we proposed a new algorithm that prioritizes the packets using IPv6 FL field. This algorithm furnishes a reliable solution to improve the QoS of IPTV sub traffic by increasing the priority of BC traffic in the detriment of VoD and PVR flows. We also applied our technique to an LTE-WLAN Heterogeneous system which gave a better quality of IPTV services. The empirical lab in Section 4 allows to predict the performance of this algorithm. The performance results reveal that in case of moving user, the topmost is for the amount of data received by $\mathrm{BC}$ user which has the highest priority. Our outcomes prove that the packet loss and end-toned delay decreased for $\mathrm{BC}$ user, but increased for PVR, which explains that our methods work well. We are working on applying this technique to the next interworking heterogeneous network (LTEWLAN-WiMAX). The security and its related issues in IPTV IMS network have always been a worry, we intend in the future to improve it and to solve these issues.

\section{Acknowledgement}

We thank Prof. Cherkaoui Leghriss for assistance with IPv6 protocol implementation and for comments that greatly improved the manuscript.

\section{Author's Contributions}

Mohamed Matoui, Noureddine Moumkine and Abdellah Adib: Contributed to the design and implementation of the research, to the analysis of the results and to the writing of the manuscript.

\section{Ethics}

This article is original and contains unpublished material. The corresponding author confirms that all of the other authors have read and approved the manuscript and no ethical issues involved. 


\section{References}

3GPP, 2011. 3G TS 23.107 V5.0.0. quality of service, concept and architecture.

Alsaffar, A., M. Aazam, C.S. Hong and E.N. Huh, 2017. An architecture of IPTV service based on PVRmicro data center and pmipv6 in cloud computing. Multimedia Tools Applic., 20: pp: 21579-21612. DOI: $10.1007 / \mathrm{s} 11042-016-4082-y$

Bhattarakosol, P., 2010. Intelligent Quality of Service Technologies and Network Management: Models for Enhancing Communication. 1st Edn., IGI Global, ISBN-10: 1615207929, pp: 404.

Conta, A. and J. Rajahalme, 2001. A model for diffserv use of the IPv6 flow label specification. IETF Internet Draft.

Deering, S. and R. Hinden, 2017. Internet protocol, version 6 (IPv6) specification, IETF Internet Draft.

Fard, H. and A. Rahbar, 2016. Physical constraint and load aware seamless handover for IPTV in wireless LANs. Comput. Electrical Eng., 56: 222-242. DOI: 10.1016/j.compeleceng.2016.01.005

Farmer, J., B. Lane, K. Bourg and W. Wang, 2016. FTTx Networks: Technology Implementation and Operation. 1st Edn., Elsevier Science, ISBN-10: 0128004584, pp: 452.

$\mathrm{Hu}, \mathrm{Q}$. and B. Carpenter, 2011. Survey of proposed use cases for the IPv6 flow label, drafthu-flowlabel-cases-03. University of Auckland.

Huang, R., X. Wei, Y. Gao, C. Lv and J. Mao et al., 2017. Data-driven QoE prediction for IPTV service. Comput. Commun., 118: 195-204. DOI: 10.1016/j.comcom.2017.11.013

Jee, R., S. Malhotra and M. Mahaveer, 2002. A modified specification for use of the IPv6 flow label for providing an efficient quality of service using a hybrid approach. IPv6 Working Group Internet Draft, Draft-banerjee-flowlabel-ipv6-qos-03.txt, Tech. Rep.

Jiang, S., 2012. Future Wireless and Optical Networks: Networking Modes and Cross-Layer Design. 1st Edn., Springer Science and Business Media, ISBN-10: 1447128222, pp: 250.

Kumar, R., R. Margolies, R. Jana, Y. Liu and S.S. Panwar, 2018. Wilitv, reducing live satellite TV costs using wireless relays. IEEE J. Selected Areas Commun., 2: 233-245.

DOI: $10.1109 /$ JSAC.2018.2804040

Leghroudi, D., M. Bellafkih, N. Moumkine and M. Ramdani, 2011. Differentiation Intra Traffic in the IPTV over IMS Context. In e-Technologies and Networks for Development. 1st Edn., Springer, pp: 329-336.
Li, M. and L. Chen, 2017. Spectrum allocation algorithms for wireless cellular networks supporting mobile IPTV. Comput. Communicat., 99: 119-127. DOI: 10.1016/j.comcom.2016.08.004

Li, M., 2017. Queueing analysis of unicast IPTV with adaptive modulation and coding in wireless cellular networks. IEEE Transact. Vehicular Technol., 66: PP: 9241-9253. DOI: 10.1109/TVT.2017.2702626

Lloret, J., A. Canovas, J. Tomas and M. Atenas, 2012. A network management algorithm and protocol for improving qoe in mobile IPTV. Comput. Communicat., 15: 1855-1870.

DOI: $10.1016 /$ j.comcom.2012.06.002

Matoui, M. N. Moumkine and A. Adib, 2017a. An IPv6 flow label based approach for IPTV quality of service. Proceedings of the International Conference on Wireless Networks and Mobile Communications, Nov. 1-4, IEEE Xplore Press, Rabat, Morocco, pp: 186. DOI: 10.1109/WINCOM.2017.8238155

Matoui, M., N. Moumkine and A. Adib, 2017b. An IPv6 flow label based approach for mobile IPTV quality of service. Proceedings of the International Conference on Innovations in Bio-Inspired Computing and Applications, (BICA' 17), Springer, Cham, pp: 69-80. DOI: 10.1007/978-3-319-76354-5_7

Matoui, M., N. Moumkine and A. Ad̄ib, 2018a. A new architecture for enhancing the QoS of IPTV video components in LTE systems. Proceedings of the International Conference on Advanced Communication Technologies and Networking, Apr. 2-4, IEEE Xplore Press, pp: 8-8.

Matoui, M., N. Moumkine and A. Adib, 2018b. IPTV quality of service improvement approach over LTE network. Int. J. Comput. Inform. Syst. Industrial Managem. Applic., 10: 272-288.

Prakash, B., 2004. Using the 20 bit flow label field in the IPv6 Header to indicate desirable quality of service on the internet. Ph.D. Thesis, Dissertation, University of Colorado.

Raouyane, B., M. Bellafkih, M. Errais and D. Ranc, 2011. Ws-composite for management and monitoring IMS network. Int. J. Next-Generat. Comput., 3: 257-270.

Sabry, E.S., R.A. Ramadan, M.A. El-Azeem and H. ElGouz, 2016. Evaluating IPTV network performance using opnet. Proceedings of the International Conference on Communication, Management and Information Technology, (MIT' 16), CRC Press, pp: 377-384.

Sambath, K., M. Abdulrahman and V. Suryani, 2016. High quality of service video conferencing over IMS. Int. J. Inform. Educ. Technol., 6: 470-476. DOI: 10.7763/IJIET.2016.V6.734 
Siddiqui, M.S., R.A. Shaikh and C.S. Hong, 2009. QoS control in service delivery in IMS. Proceedings of the 11th International Conference on Advanced Communication Technology, Feb. 15-18, IEEE Xplore Press, Phoenix Park, South Korea pp: 157-157.

Solera, M., M. Toril, I. Palomo, G. Gomez and J. Poncelan, 2018. A testbed for evaluating video streaming services in LTE, Wireless Personal Commun., 3: 2753-2773.

DOI: $10.1007 / \mathrm{s} 11277-017-4999-0$
Tsolkas, D., E. Liotou, N. Passas and L. Merakos, 2017. A survey on parametric QoE estimation for popular services. J. Network Comput. Applic., 77: 117-117. DOI: 10.1016/j.jnca.2016.10.016

Zamora, D.C. and H. Przybysz, 2011. Policy and charging control architecture. uS Patent App. 